\title{
Recht, Politik und die Bedingungen der Ko-Präsenz: Das „Recht, Rechte zu haben“" im Lichte gegenwärtiger Migrationsfragen
}

\author{
Dana Schmalz
}

\begin{abstract}
Hannah Arendt's expression of the "right to have right" forms a focal point in debates about the foundation of rights and the possibility to prevent rightlessness. Not only in Arendt's text but also in later scholarship that draws on the expression, it is particularly the situation of migrants that drives the reflections. However, the role of spatial distance for rights has received surprisingly little attention. The paper explores how spatial distance relates to legal guarantees and affects the claiming of rights. It discusses the different interpretations that the "right to have rights" was given and asks about its persistent relevance in times of international human rights treaties. Nowhere is the precarity of the "right to have rights" today as palpable as in the conditions of accessing territory in search of protection. The reach of fundamental rights guarantees regularly hinges on the jurisdiction of a state, laws and practices of hindering access can easily circumvent legal liability. And even where the legality is in doubt, the physical distance of potential claimants leaves most cases unchallenged. The paper revisits the debate about the "right to have rights" with view to the situation of distant claimants. It argues that theories of political contestation often build on the co-presence of persons as an implicit premise and that this gives rise to a puzzle since co-presence is itself conditioned by legal rules. The paper suggests that this requires us to examine how conditions of presence, visibility and communication affect the political founding and negotiation of rights, and to ask how law shapes these conditions.
\end{abstract}

Keywords: Hannah Arendt, Human Rights, Migration, Rightlessness, Territoriality, Universal Rights

\section{Weshalb Rechte?}

Migration wird heute wesentlich im Vokabular von Rechten diskutiert. Vor dem Europäischen Gerichtshof für Menschenrechte oder vom Menschenrechtskomitee der Vereinten Nationen wird verhandelt, welches staatliche Handeln hinsichtlich Migration zulässig oder geboten ist - anhand der Rechte von Migrierenden. Die Entwicklungen in den Migrationsbewegungen in Europa der letzten Jahre - wo Menschen bleiben dürfen, wann sie Freizügigkeit genießen, wann diese ihnen verwehrt wird - gehen wesentlich auf das Festschreiben und das gerichtliche Auslegen von Rechtspositionen zurück. Man denke nur daran, unter welchen Bedingungen Dublin-Überstellungen zulässig sind: Diese Frage war anfangs objektiv-rechtlich, also ohne individuelle Rechtspositionen, geregelt. Die Regelungen wurden dann aber bald von menschenrechtlichen Positionen 
überlagert, ${ }^{1}$ und schließlich fanden subjektive Rechte Eingang in die Dublin-Verordnung. ${ }^{2}$ Viele andere Bereiche lassen sich anführen. ${ }^{3}$ Migration ist nicht nur rechtlich eine Frage von Rechten, sondern wird auch politisch weitgehend als solche verhandelt. ${ }^{4}$

Nicht nur ist das Vokabular von Rechten nicht aus den Migrationsdebatten wegzudenken, Migration bildet umgekehrt auch ein Feld, in dem die Grundfragen nach der Natur von Rechten unumgänglich sind. Sicher, in vielen gesellschaftlichen Bereichen wird über Inhalt und Reichweite von Rechten verhandelt. Was den Bereich der Migration unterscheidet, ist der inhärente Bezug auf Territorium und auf politische Zugehörigkeit, beides Kategorien, welche die Grundlage von Rechten betreffen. Das Territorium bestimmt ganz wesentlich den Geltungsraum von Rechten. Die politische Zugehörigkeit wiederum bildet, in einem demokratischen System, die Legitimationsgrundlage von Rechten als Ergebnis demokratischer Rechtsetzungsprozesse. Territorium und politische Zugehörigkeit sind im Territorialstaat aufeinander bezogen, und Migration betrifft zunächst den territorialen Zugang und potentiell, bei dauerhafter Anwesenheit, auch den Zugang zu politischer Zugehörigkeit. Es sind auch diese Verknüpfungen, die Migration zu einem Thema von so großer politischer Tragweite machen. Und diese Verbindung macht Migration interessant für die Frage von Rechten.

Im Kontext von Migration geht es in besonderer Weise um Grundlage und Begründung von Rechten. Wenn auf dem Mittelmeer ein Boot in Seenot gerät und Italien auf Anrufe hin sich auf seine fehlende Rettungszuständigkeit beruft und an Maltas Seenotrettungskoordination verweist: handelt es sich dabei um eine Rechtsverletzung? ${ }^{5}$ Wenn in der belgischen Botschaft im Libanon eine syrische Familie ein Visum zur Einreise beantragt und dieses verweigert wird trotz unmenschlicher Bedingungen, denen die Familie im Libanon oder Syrien ausgesetzt ist: handelt es sich dabei um eine Rechtsverletzung $?^{6}$ In diesen Fällen der letzten Jahre, ebenso wie in zahlreichen weiteren, ging es um sehr grundlegende Fragen nach dem Wesen von Rechten. Zunächst steht bei den Fällen die Reichweite von staatlicher Verantwortung für Rechte zur Debatte. Auch wenn dies die Auslegung von rechtlichen Vorgaben, beispielsweise von Art. 1 EMRK, erfordert, so stützt sich diese Auslegung notwendigerweise auf breitere theoretische Überlegungen, die von dem Wortlaut der Norm nicht vorgegeben sind. Wann befinden sich Personen unter der Hoheitsgewalt eines Staates? Bei der Auslegung spielen Verständnisse von schlüssiger Verantwortung eine überragende Rolle, wie auch die entsprechenden Rechtsprechungspassagen verdeutlichen. ${ }^{7}$

${ }^{1}$ S. EGMR (Große Kammer), Urteil vom 21.1.2011 - 30696/09 (M.S.S. u. a. gegen Belgien und Griechenland).

${ }^{2}$ So in den Urteilen Ghezelbash, EuGH, Urteil vom 7.6.2016, ECLI:EU:C:2016:409; Mengesteab, EuGH, Urteil vom 26.7.2017, ECLI:EU:C:2017:587. Dazu auch Schmalz, Subjektive Rechte aus der Dublin-Verordnung: Der Fall Mengesteab vor dem EuGH, Verfassungsblog 29.7.2017, https://verfassungsblog.de/subjektive-rechte-aus-der-dublin-verordnung-der-fall-mengesteab-vor-dem-eugh/

${ }^{3}$ Vgl. etwa EGMR, Urteil vom 28.2.2019 - 12267/16 (Khan gegen Frankreich).

${ }^{4}$ Das Deutsche lässt diese Aussagen nahezu tautologisch erscheinen, weil das Recht (als objektives) und Rechte (als subjektive) quasi wortgleich sind. Die unterschiedlichen Begriffe in anderen Sprachen (z. B. law - rights) können verdeutlichen, dass es sich keineswegs um Tautologien handelt.

${ }^{5}$ So in UN Menschenrechtskomitee, Entscheidung vom 27.1.2021, CCPR/C/130/D/3042/2017.

${ }^{6}$ So diskutiert in den Fällen EuGH, X und X, Urteil vom 7.2.2017, C-638/16 und EGMR (Große Kammer), Urteil vom 5.5.2020 - 3599/18 (M.N. u. a. gegen Belgien).

${ }^{7}$ Vgl. EGMR (Große Kammer), Urteil vom 5.5.2020 - 3599/18 (M.N. u. a. gegen Belgien), $\S \S 110$ ff.; vgl. auch EGMR (Große Kammer), Urteil vom 13.2.2020 - 8675/15 und 8697/15 (N.D. und N.T. gegen Spanien), $§ 110$. 
Die Reichweite der Verpflichtungen von Staaten wird dabei im Lichte des Universalitätsanspruchs von Menschenrechten ausgelegt. Das Gegenüber von universellen Rechten und begrenzter staatlicher Verantwortung für diese Rechtsgarantien steht in einem ständigen Wechselspiel der Auslegung. Rechte bestehen nur insoweit tatsächlich, als ihnen auch Pflichten zur Wahrung dieser Rechte entsprechen. Diese Pflichten können ihrerseits nicht blind für die Idee universeller, also für alle garantierter, Rechte sein - die Vermeidung von Rechtlosigkeit leitet die Auslegung der Rechtsnormen als ein Argument. Doch die Fragen nach der Grundlage von Rechten stellen sich ganz unmittelbar nicht nur bei gerichtlichen Verfahren. Die gerichtliche Bestimmung von Rechten erfolgt immer nachträglich, als Konstatieren der Verletzung, insofern gewissermaßen immer ,zu spät" ${ }^{8}$ Migrationskonstellationen werfen in vieler Hinsicht die Frage nach Rechten in Unabhängigkeit von ihrer gerichtlichen Anerkennung auf: Was bedeutet es für Rechte, dass ihre potentiellen Träger:innen selten in der Lage sind, überhaupt ein Verfahren zur Prüfung anzustellen?

Rechte sind nicht existent oder nicht-existent, sie sind oftmals umstritten. Emanzipatorische Bewegungen erstreiten Rechte als positive Rechte, die sie zuvor nicht haben. Insofern wird das Vokabular von Rechten teilweise gerade im Kontrast zu bereits im positiven Recht anerkannten Rechten verwendet. Umgekehrt lässt sich die Frage stellen, ob man ein Recht „hat“, wenn es zwar formal niedergeschrieben ist, aber nicht tatsächlich respektiert wird. Rechte, wie zu betrachten sein wird, entstehen und bestehen ganz wesentlich durch Anerkennung. Doch diese Prozesse des Erstreitens von Rechten sind selbst durch rechtliche Vorgaben geprägt. In dem Wechselspiel von Recht und Politik hinsichtlich der Anerkennung von individuellen Rechten bildet wiederum Migration ein besonderes Feld.

Aus dem akademischen, aber auch dem politischen Gespräch über die Ränder und die Voraussetzung von Rechten ist Hannah Arendts Ausdruck des „Rechts, Rechte zu haben" nicht mehr wegzudenken. Dabei ist das erst spät einsetzende und heute enorme Interesse an dem Ausdruck selbst bemerkenswert. . Arendt verwendet den Ausdruck erstmals 1949 in einem Aufsatz ${ }^{10}$ und dann ausführlicher in ihrem 1951 auf englisch, 1955 auf deutsch erschienenen Buch Elemente und Ursprünge totaler Herrschaft ${ }^{11}$ im Kapitel zu den Aporien der Menschenrechte. Erst in den 1990er Jahren wird der Ausdruck des „Rechts, Rechte zu haben“ prominent aufgegriffen. ${ }^{12}$ Das kann als Aspekt

\footnotetext{
${ }^{8}$ Vgl. den Schlussantrag des Generalanwalts Mengozzi in EuGH, X und X, 7.2.2017, C-638/16, $\S 166$.

${ }^{9}$ So auch DeGooyer, The Right to Have Rights, in: DeGooyer u. a. (Hrsg.), The Right to Have Rights, 2018, 10.

${ }^{10}$ Arendt, The Rights of Man: What are They?, Modern Review 3/1 (1949), 24 (30).

${ }^{11}$ Arendt, Elemente und Ursprünge totaler Herrschaft (1955), hier im Folgenden zitiert von der Ausgabe Piper Verlag München, 17. Aufl. 2014.

${ }^{12}$ Erste Bezugnahmen finden sich in King, Endings and Beginnings: Politics in Arendt's Early Thought, Political Theory 12/2 (1984), 235; Aleinikoff, Theories of Loss of Citizenship, Michigan Law Review 84/7 (1986), 1471; Benhabib, Hannah Arendt and the Redemptive Power of Narrative, Social Research 57/1 (1990), 167. Ausführlichere Auseinandersetzungen finden sich dann bei Michelman, Parsing “A Right to Have Rights", Constellations 3/2 (1996), 200; Isaac, A New Guarantee on Earth: Hannah Arendt on Human Dignity and the Politics of Human Rights, The American Political Science Review 90/1 (1996), 61.
} 
eines generell zunehmenden Interesses an Hannah Arendts Werk gewertet werden, ${ }^{13}$ aber es spiegelt auch wider, dass der Ausdruck einen Ansatzpunkt für strukturelle Fragen von Menschenrechten wurde, welche sich in dieser Phase immer stärker stellten. Das fasst Ayten Gündoğdu in ihrem Buch Rightlessness in an Age of Rights. Hannah Arendt and the Contemporary Struggles of Migrants von 2015 so zusammen: Was bedeutet es rechtlos zu sein in einem Zeitalter der Menschenrechtsabkommen und einer Allgegenwärtigkeit des Rechte-Vokabulars im internationalen Diskurs? ${ }^{14}$

Die rahmende Situation von Grund- und Menschenrechten hat sich erheblich verändert gegenüber dem Zeitpunkt, in dem Arendt den Ausdruck des „Rechts, Rechte zu haben" formulierte. Doch in der spezifischen Menschenrechtskritik, an welche er anschließt, und in seiner Einbettung in Arendts Denken, welches das Zusammenspiel von Recht und Politik in besonderer Weise zum Gegenstand hat, ${ }^{15}$ entpuppt sich der Ausdruck als fruchtbarer Ankerpunkt für heutige Debatten.

Der vorliegende Beitrag betrachtet die Weise, in der das „Recht, Rechte zu haben“ in den vergangenen Jahren in Bezug auf Migration interpretiert wurde, und argumentiert, dass Konstellationen der physischen Distanz bei der Debatte um Rechte und Rechtsbegründung stärker berücksichtigt werden sollten. Der nächste Abschnitt betrachtet, wie die Formel bei Arendt selbst eingeführt wird und wie sie an eine Kritik von Menschenrechten anschließt (II.). Der darauffolgende dritte Abschnitt zeichnet dann Interpretationen nach, die auf eine geänderte rahmende Rechtslage mit inzwischen weitreichend kodifizierten und gerichtlich überprüfbaren Menschenrechten reagieren (III.). Ein vierter Abschnitt blickt auf den Fokus auf politische Kämpfe als Grundlage des „Rechts, Rechte zu haben“, wie er besonders von und im Anschluss an Jacques Rancière formuliert wurde (IV.). Der fünfte Abschnitt erörtert daran anschließend, wie sich das Zusammenspiel von Recht und Politik bei der Begründung von Rechten in Konstellationen physischer Distanz, also in vielen Migrationsfragen, verändert (V.). Der Beitrag schließt mit einem Blick auf die Rolle von Kopräsenz für Recht und Politik (VI.).

\section{Kritik der Menschenrechte und das notwendige ,Recht, Rechte zu haben“6}

Wenn heute über das „Rechte, Rechte zu haben“ gesprochen wird, dann geht es um Situationen, in welchen grundlegende Rechte verwehrt werden. Interesse der Formel ist die letzte Absicherung von Rechten, sie sucht nach der Garantie gegen Rechtlosigkeit. So steht der Ausdruck unmittelbar in der Denkgeschichte von Menschenrechten, also der Idee, es müsse Rechte geben, welche ohne weitere Bedingungen, einfach qua Menschsein garantiert sind. Doch wenn Hannah Arendt das „Recht, Rechte zu haben“ in ,Elemente und Ursprünge totaler Herrschaft“ einführt, dann geschieht das unmittelbar nach einer nachdrücklichen Kritik an Menschenrechten. Diese Kritik ist wesentlich für das Verständnis des Rechts, Rechte zu haben.

\footnotetext{
${ }^{13}$ In diesem Zusammenhang sei nur auf die wohl einflussreiche Werkbiographie Arendts hingewiesen, die ebenfalls in diesen Jahren erschien: Benhabib, The Reluctant Modernism of Hannah Arendt (1996).

${ }^{14} \mathrm{Vgl}$. Gündoğdu, Rightlessness in an Age of Rights. Hannah Arendt and the Contemporary Struggles of Migrants, 2015, 11 .

${ }^{15}$ Dazu besonders Volk, Arendtian Constitutionalism. Law, Politics and the Order of Freedom, 2015, 173.
} 
Die Idee universeller Rechte bekam einen prominenten Platz im politischen Denken mit der französischen Erklärung der Menschen- und Bürgerrechte 1789. Gemeinsam mit der Entwicklung des territorialen und später demokratischen Staates bildete sich die Rolle individueller Rechte heraus. In dieser neuen Konzeption von Legitimität stand das Individuum im Zentrum bzw. am Anfang, wie die Theorien des Gesellschaftsvertrags verdeutlichen. Einerseits sind also die universalistischen Werte menschlicher Gleichheit und Freiheit grundlegend für das Legitimitätsdenken der Moderne. Andererseits entstehen die Institutionen des modernen Staats innerhalb territorialer Grenzen. Gleichheit und Freiheit werden insofern durch partikulare Institutionen und Rechtspositionen garantiert: durch das Institut der Staatsbürgerschaft, welche sich am Staatsterritorium orientiert, aber auch durch Rechtsgarantien, die vorrangig auf dem Territorium gelten. Für so genannte universelle Rechte wirft das die Frage auf, ob sie mehr als eine moralische Idee sind. Wenn Recht innerhalb des Staates geschaffen wird, woher beziehen dann universelle Rechte ihre Kraft oder ihre Geltung?

Ein solcher, gewissermaßen positivistischer, Einwand gegen die Bedeutung von Menschenrechten hätte sich inzwischen weitgehend erübrigt. Heute lässt sich anführen, dass von den Menschenrechtspakten von 1966, ${ }^{16}$ über regionale Menschenrechtsabkommen bis zu themenspezifischen Abkommen die Menschenrechte auch jenseits des Staates kodifiziert sind. Arendts Kritik setzt allerdings anders an: Das Problem der Menschenrechte war gerade, dass sie sich auf einen „Menschen überhaupt“ $z$ u beziehen behaupteten, den es so nie gab. ${ }^{17}$ Zusammen mit der Idee, dass sie voraussetzungslos und unveräußerlich sein sollten, beruhten Menschenrechte in diesem Sinne auf der Illusion, es gäbe Menschen außerhalb sozialer und politischer Kontexte. Wie schwach diese Idee von Rechten des gänzlich abstrakten Menschen war, zeigte sich in der praktischen Wirkungslosigkeit menschenrechtlicher Ideen, als Minderheiten in Europa verfolgt wurden. ${ }^{18}$ Praktisch bestand kein Schutz für diejenigen, deren Rechte nicht durch einen Staat geschützt wurden. ${ }^{19}$ Dabei geht es nicht lediglich um einen Mangel an Durchsetzbarkeit, sondern um einen inhärenten Widerspruch: Rechte bestehen zwischen Menschen, sie beruhen auf der gegenseitigen Ansehung und Anerkennung. Insofern ist es unsinnig, von Rechten eines Menschen jenseits dieser zwischenmenschlichen Existenz auszugehen.

Dass Menschen immer ,im Plural“" existieren, ist ein zentraler Aspekt von Arendts Denken, den sie auch in ihrem späteren Werk „Vita activa“ ausführt. ${ }^{20}$ Ihre Kritik am Konzept der Menschenrechte hängt nicht an deren rechtlicher Stellung, und hat sich insofern nicht mit der Kodifizierung erübrigt. Vielmehr weist sie auf den Zusammenhang zwischen Rechten und der Anerkennung von Menschen als politische Wesen hin. Weil Menschen immer in Zusammenhängen leben, können sie Rechte als effektive Rechte nur haben, wenn sie auch in diesen Zusammenhängen gesehen werden - also als Personen, die in ihrer Erfahrung der Welt anderen begegnen, Meinungen ausbilden und mit Handlungen in die Welt eingreifen. „Der Verlust der Menschenrechte“, schreibt Arendt, findet statt,

\footnotetext{
${ }^{16}$ Internationaler Pakt über bürgerliche und politische Rechte (IPbpR) und Internationaler Pakt über wirtschaftliche, soziale und kulturelle Rechte (IPwskR) vom 19. Dezember 1966.

${ }^{17}$ Arendt, Elemente und Ursprünge totaler Herrschaft, 17. Aufl. 2014, 604.

${ }^{18}$ Ibid., 605.

${ }^{19}$ Ibid., 606.

${ }^{20}$ Arendt, Vita activa oder vom tätigen Leben (1967), hier zitiert aus der Ausgabe Piper Verlag München, 10. Aufl. 2011, 12, 213.
} 
„wenn der Mensch den Standort in der Welt verliert, durch den allein er überhaupt Rechte haben kann und der die Bedingung dafür bildet, dass seine Meinungen Gewicht haben und seine Handlungen von Belang sind. “21

Vor diesem Hintergrund führt Arendt den Ausdruck des „Rechts, Rechte zu haben“ ein. Sie schreibt:

\footnotetext{
„Dass es so etwas gibt wie ein Recht, Rechte zu haben - und dies ist gleichbedeutend damit, in einem Beziehungssystem zu leben, in dem man aufgrund von Handlungen und Meinungen beurteilt wird -, wissen wir erst, seitdem Millionen von Menschen aufgetaucht sind, die dieses Recht verloren haben und zufolge der neuen globalen Organisation der Welt nicht imstande sind, es wiederzugewinnen." 22
}

Diese Aussage kann überraschen nach der vorangegangenen Kritik: wenn Rechte gerade nicht außerhalb von politischer Anerkennung wirksam existieren, was meint dann ein „Recht, Rechte zu haben“? Unterliegt dieses Recht nicht denselben Schwierigkeiten, denen Menschenrechte unterliegen?

Das „Recht, Recht zu haben“ scheint insofern eine Frage eher als eine Antwort zu sein. Es ist der Hinweis auf eine Notwendigkeit für ein solches Recht, eingeführt in der Verneinung: Wir erkennen die Notwendigkeit eines solchen Rechts aus seinem Verlust oder seinem Fehlen. Das ist nicht so anders als die Struktur von Rechten allgemein: Wir bezeichnen als Rechte die gegenseitig anerkannten Handlungsspielräume und Existenzmöglichkeiten, die teilweise in Frage stehen oder verletzt werden. Nicht alles, was Menschen tun können, korrespondiert mit einem ausdrücklichen Recht - so sprechen wir nicht von einem „Recht, zu atmen“ oder einem „Recht, Kleidung zu tragen“, auch wenn diese unter die allgemeine Handlungsfreiheit gefasst werden können; sie sind üblicherweise aber nicht bestritten. Ausdrückliche Rechtspositionen sind insofern ein Spiegel gesellschaftlichen Streits oder fehlender gesellschaftlicher Unterstützung. ${ }^{23}$ Rechte kommen dort ins Spiel, wo ihre Verletzung im Raum steht. ${ }^{24}$

Das „Recht, Rechte zu haben“ stellt Arendt also als eine Notwendigkeit in den Raum. Dass dieses Recht kein einfaches Recht ist, ist nach dem Vorangegangenen klar. Vielmehr bringt die Formel eine Suche nach der minimalen Garantie von Rechten auf den Punkt: Wie lässt sich vermeiden, dass Menschen gänzlich aus dem Schutz durch Recht herausfallen? Der Ausdruck rückt den Scheinwerfer auf den Zusammenhang von Recht und Politik in der Begründung und Absicherung von Rechten.

\footnotetext{
${ }^{21}$ Arendt, Elemente und Ursprünge totaler Herrschaft, 17. Aufl. 2014, 613.

${ }^{22}$ Ibid., 614.

${ }^{23}$ Interessant in diesem Zusammenhang sind auch Sprachenrechte, die nur für sprachliche Minderheiten in politisch angespannten Situationen ein ansonsten sozial unumstrittenes Verhalten zum Gegenstand rechtlicher Auseinandersetzungen machen. Dazu Schmalz, Beyond an Anxiety Logic: A Critical Examination of Language Rights Cases Before the European Court of Human Rights, Human Rights Law Review 20/1 (2020), 101.

${ }^{24}$ Vgl. auch generell zu der Möglichkeit des Bruchs als Gehalt jeder Normierung Möllers, Die Möglichkeit der Normen, 2015, 153.
} 


\section{Das „Recht, Rechte zu haben“6 im Zeitalter von Menschenrechtsabkommen}

Die Bedingungen von universellen Rechten haben sich in den letzten 70 Jahren erheblich verändert. Das „Recht, Rechte zu haben“" wurde vielfach im Lichte dieser neuen Rechtslage interpretiert. Dabei ist der Blick auf zwei Bereiche rechtlicher Entwicklungen zu richten: Zum einen kodifizieren internationale Menschenrechtsabkommen Rechte jenseits des Staates. Der Universalitätsanspruch von grundlegenden Rechten und die Grenzen dieser Universalität sind hier neu zu betrachten. Zum anderen besteht heute mit dem internationalen Flüchtlingsrecht ein Recht, dass sich auf Garantien für diejenigen richtet, deren Rechtlosigkeit Arendt beschrieb.

Verschiedene Beiträge besonders aus den Rechtswissenschaften haben in durchaus anspruchsvoller Weise die Formel des „Rechts, Rechte zu haben“ auf neue Rechtsgarantien bezogen. So liest Nanda Oudejans das „Recht, Rechte zu haben“ in der gegenwärtigen Zeit gerade als Recht auf Asyl: Der „Verlust des Ortes“ und damit der politischen Zugehörigkeit, welche die Situation von Flüchtlingen kennzeichne, entspreche dieser Rechtlosigkeit, welche Arendt beschreibe, und so sei das Recht auf Asyl die nächste rechtliche Antwort auf diesen Verlust. ${ }^{25}$ Alison Kesby untersucht den Ausdruck des „Rechts, Rechte zu haben“ entlang zweier Achsen, der Achse des Ortes und der Achse des Subjekts. ${ }^{26}$ Wenn Arendt über den ,Platz in der Welt“" spricht, ${ }^{27}$ welcher beim Verlust der Rechte auf dem Spiel steht, ist nicht lediglich der physische Raum, sondern die Existenz als politisches Wesen gemeint. Einen Platz in der Welt zu haben, bedeutet in diesem Sinne, an der Welt teilzunehmen, welche Menschen gemeinsam durch Handeln erzeugen. Das „Recht, Rechte zu haben“ ist in Kesbys Interpretation zunächst die Zuerkennung eines solchen Platzes in der Welt. ${ }^{28}$ Die Bedeutung dessen entwickelt sie entlang von vier Aspekten: Staatsangehörigkeit, Bürgerschaft, Menschheit und Politik. Das Recht auf Staatsangehörigkeit bildet in dieser Lesart eine Dimension des „Rechts, Rechte zu haben“" als die solideste Garantie von Rechten. Im Fall von Flüchtlingen wird aber auch deutlich, dass eine formale Staatsangehörigkeit nicht zwingend schützt. Zur Menschheit zu gehören wiederum, bedeutet keinerlei konkrete Garantie; der Bezug darauf dient allenfalls aber als Aspekt des Erstreitens von Rechten. ${ }^{29}$

Diese Lesarten, welche das Recht auf Asyl, auf Staatsangehörigkeit oder auf politische Rechte in den Blick nehmen, buchstabieren allesamt wichtige Aspekte in der Bedingung von sicheren Rechten aus. Dennoch ist der Ausdruck des „Rechts, Rechte zu haben“ mehr als der Hinweis auf spezifische notwendige Rechte. Der Ausdruck bringt einen Knoten zum Ausdruck, welcher sich so leicht nicht lösen lässt. Umgekehrt erklärt es die anhaltende Wirkkraft des Ausdrucks, dass er mehr zusammenfasst als ein zu garantierendes Recht.

Es lohnt, genauer zu betrachten, weshalb heute trotz weitreichender Garantien im positiven Recht die Frage nach dem „Recht, Rechte zu haben“ nicht erledigt scheint. Mit internationalen Menschenrechtsabkommen bestehen jenseits von Staten Rechts-

\footnotetext{
${ }^{25}$ Oudejans, The Right to Have Rights as the Right to Asylum, Netherland Journal of Legal Philosophy 43/1 (2014), 7 (11).

${ }^{26}$ Kesby, The Right to Have Rights: Citizenship, Humanity, and International Law, 2012, 6.

${ }^{27}$ Arendt, Elemente und Ursprünge totaler Herrschaft, 17. Aufl. 2014, 607.

${ }^{28}$ Kesby, The Right to Have Rights: Citizenship, Humanity, and International Law, 2012, 13.

${ }^{29}$ Ibid., 117.
} 
garantien, die teilweise durch eigene Spruchkörper wie die Menschenrechtskommission, den europäischen Gerichtshof für Menschenrechte oder den interamerikanischen Gerichtshof für Menschenrechte ausgelegt und konkretisiert werden. Die darin niedergelegten Rechte gelten universell, insofern sie Menschen unabhängig von Staatsangehörigkeit schützen. Dennoch wird gerade im Kontext von Migration auch immer wieder die Begrenztheit dieser Garantien deutlich. Wenn Menschen fliehen müssen, aber keine Zuflucht finden und kein Staat rechtlich für sie verantwortlich ist, so kann dies als Rechtlosigkeit bezeichnet werden. ${ }^{30}$

Es gibt verschiedene Gründe, weshalb Rechte gerade für Flüchtende prekär bleiben. Der Erste ist die rechtliche Reichweite von Garantien. Meist ist die Ankunft auf dem Territorium für Asylsuchende gerade die Voraussetzung, um Schutz zu erhalten. Zugleich ist die staatliche Verantwortung für Menschenrechte im Ausgangspunkt territorial. Die Garantien universeller Rechte sind nur insoweit von tatsächlichem Nutzen für Menschen, wie sie sich auch gegenüber einem Staat darauf berufen können. Die Begrenzung der staatlichen Verantwortungsbereiche ist erst einmal sinnvoll: nicht jeder Staat hat gegenüber jedem Menschen eine Verantwortung unter Menschenrechtsabkommen. Zugleich führen diese Abgrenzungen im Bereich von Migration leicht in rechtliche Leerstellen oder zumindest Grauzonen der Verantwortung. ${ }^{31}$

Für die EMRK legt Art. 1 die Anwendbarkeit fest: Staaten sind gegenüber ,allen ihrer Hoheitsgewalt unterstehenden Personen“" zur Sicherung der Rechte und Freiheiten aus der Konvention verpflichtet. Hoheitsgewalt besteht grundsätzlich auf dem Territorium; in besonderen Fällen wird extraterritoriale Hoheitsgewalt angenommen, wenn ein Staat „effektive Kontrolle“ über Personen ausübt. ${ }^{32}$ Diese effektive Kontrolle wurde überwiegend als physische Kontrolle ausgelegt. ${ }^{33}$ Als solche führt die Schwelle aber gerade im Fall von Migration oft zu Schwierigkeiten. Staatliche Praktiken, die Personen vom Erreichen des Territoriums und damit eines Schutzanspruchs abhalten wollen, finden regelmäßig gerade in diesem Grenzbereich statt, wie in den eingangs erwähnten Beispielen: auf Schiffen, in Form von Kooperation mit Drittstaaten, unter Einbeziehungen von privaten Akteuren. So mag die Verwehrung von Schutz nicht unbedingt als Rechtsverletzung unter einem Abkommen gelten. Besonders wenn Menschen in jedem Staat ungewollt sind und durch Praktiken der Zurückweisung nirgendwo in Sicherheit kommen können, entleeren sich Rechtsgarantien aber wie das Recht auf Leben und auf Unversehrtheit der Person. Ein erster Aspekt, weshalb Rechtsgarantien in internationalen Abkommen also nicht das Problem des „Rechts, Rechte zu haben“ aufheben, ist das Zusammenspiel von Universalitätsanspruch der Rechte einerseits und begrenzter Verantwortung andererseits.

${ }^{30}$ Vgl. Mann, Maritime Legal Black Holes: Migration and Rightlessness in International Law, EJIL 29/2 (2018), 347; vgl. auch im Schlussantrag des Generalanwalts Mengozzi in EuGH, X und X, 7.2.2017, C-638/16, § 157 .

${ }^{31}$ Dazu auch Schmalz, Zur Reichweite von Menschenrechtspflichten: Zugang zu Schutz an den Grenzen Europas, Newsletter Menschenrechte 2019/5, 367.

${ }^{32}$ EGMR (Große Kammer), Urteil vom 12.12.2001 - 52207/99 (Bankovic u. a. gegen Belgien), $\S 71$; Tzevelekos, Reconstructing the Effective Control Criterion in Extraterritorial Human Rights Breaches: Direct Attribution of Wrongfulness, Due Diligence, and Concurrent Responsibility, Michigan Journal of International Law 36/1 (2014), 129.

${ }^{33}$ EGMR (Große Kammer), Entscheidung vom 23.2.2012 - 27765/09 (Hirsi Jamaa u. a. gegen Italien), § 81; vgl. auch $\mathrm{Paz}$, Between the Kingdom and the Desert Sun: Human Rights, Immigration, and Border Walls, Berkeley Journal of International Law 34/1 (2016), 17. 
Ein zweiter Aspekt, weshalb Rechte gerade für Migrierende oft prekär bleiben, ist die praktische Schwierigkeit, sie einzuklagen. Menschenrechtsgarantien, selbst dort wo sie formal gelten, sind häufig praktisch nicht erreichbar für diejenigen, die durch Praktiken der Zurückweisung betroffen sind und sich dann in Distanz befinden. Viele weitere Faktoren könnten aufgezählt werden: von der Hürde das Rechtssystem zu überblicken, über finanzielle Hürden, bis hin zu den Sorgen, mit einer Klage dem eigenen Schutzanspruch zu schaden. In diesen Fällen ist die Frage, ob Rechte bestehen, zunächst offen - sie müsste erst in Verfahren geklärt werden. Doch zu diesen Verfahren kommt es meist nicht. Durch all dies bleibt die Idee universeller Rechte auch heute noch vielfach ein uneingelöstes Versprechen.

\section{Politische Kämpfe als Begründung von Rechten}

Um dem Aussagegehalt des „Rechts, Rechte zu haben“ näher zu kommen, ist der Blick über juridische Garantien hinaus notwendig. Verschiedene Interpretationen haben den Ausdruck auf das Verhältnis von moralischen Ansprüchen, rechtlichen Festschreibungen und politischen Kämpfen bezogen.

Seyla Benhabib liest in ihrer einflussreichen Interpretation des „Rechts, Rechte zu haben" Arendt mit Immanuel Kant zusammen. ${ }^{34}$ Sie unterscheidet zwei Arten von Rechten: Das Recht, Rechte zu haben, spiegelt ein moralisches Recht auf minimale politische Zugehörigkeit - als Anspruch, welcher sich an die Menschheit richtet. Die Rechte, die zu haben jeder ein Recht hat, hingegen beziehen sich auf die juridischen Rechte innerhalb einer Gemeinschaft. ${ }^{35}$ Die beiden Verwendungen von "Recht" bzw. "Rechten" haben insofern unterschiedliche Qualität, einmal als juridische Rechte, und einmal als moralischer Anspruch. Benhabib bezieht sich dabei auf Überlegungen von Kant zu dem „Recht der Menschheit“ in jeder Person, also der Idee, dass sich aus dem kategorischen Imperativ, niemanden je ,bloß als Mittel“ zu behandeln auch die minimalen Pflichten gegenüber Fremden ableiten lassen. ${ }^{36}$

Davon ausgehend verweist Benhabib auf das Potential der Sphäre jenseits des Staats für die Garantie von Rechten und politischer Zugehörigkeit. Diese Lesart wurde teilweise als allzu optimistisch bewertet. ${ }^{37}$ Doch man kann anerkennen, dass internationale Abkommen und Gerichte Räume bilden, in denen die bestehenden Rechte immer wieder auch in Bezugnahme auf den moralischen Anspruch auf Rechte aufgerufen und verhandelt werden können, ohne dass man damit das Problem des „Rechts, Rechte zu haben“ für erledigt erklärt. Es sind insofern nicht lediglich die rechtlichen Garantien jenseits des Staates, sondern auch die Räume für rechtliche und moralische Verhandlung, welche eine Annäherung an das „Recht, Rechte zu haben“ versprechen.

Benhabib richtet das Augenmerk insofern auf die Kraft moralischer Argumente, also eines moralischen Menschenrechtsvokabulars, welches in politischen ebenso wie in rechtlichen Prozessen artikuliert wird. Mit anderem Schwerpunkt, aber letztlich ähnlichen Annahmen, haben verschiedene Autor:innen die Rolle politischer Prozesse betont, in denen Rechte begründet und ihre Reichweite und Bedeutung verhandelt wer-

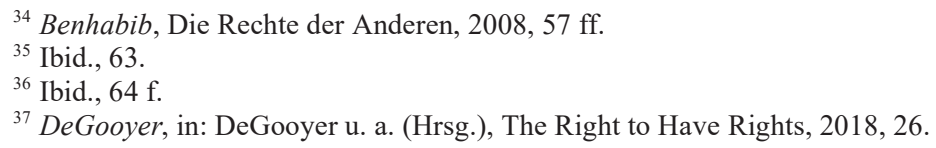


den. Rechte sind nicht statisch, ihre Auslegung bleibt immer ein Stück weit offen. Sie beruhen nicht auf einer vorpolitischen Gegebenheit, sondern sind die Ergebnisse von kollektivem politischem Handeln und gegenseitiger Anerkennung. Diese Prozesse von politischer Auseinandersetzung lassen sich einerseits hinsichtlich der verwendeten moralischen Argumente, andererseits als Konfrontationen beschreiben. Diese Perspektiven schließen sich nicht aus, weisen aber in unterschiedliche Richtungen der Analyse.

Arendt schärft mit ihrer Kritik der Menschenrechtsrhetorik zunächst den Blick dafür, dass Rechte nicht bestehen, indem sie als solche bezeichnet werden. Sie beruhen auf einem Wechselspiel von Anerkennung und Einforderung: Rechte mögen verletzt werden, ohne dass damit ihre Qualität als Rechte verloren geht; aber ohne ein Minimum an Anerkennung, dass eine Person potentielle Rechtsträgerin ist, lässt sich schwerlich von Rechten sprechen. Insofern lässt sich an Arendt eine Skepsis gegenüber moralischem Menschenrechtsvokabular anschließen, insofern die so bezeichneten Rechte als gegeben vorausgesetzt werden. Das heißt umgekehrt nicht, dass ein moralisches Argument, welches Rechte durch Bezug auf das gemeinsame Menschsein und die geteilte Welt einfordert, als solches ungeeignet sei.

Zugleich bedeutet der Streit um Rechte, dass bislang nicht anerkannte Rechtsträger Rechte einfordern und in Anspruch nehmen. Mehrere Autor:innen haben das „Recht, Rechte zu haben" in den letzten Jahren gerade auf die Bedingungen bezogen, unter welchen Rechte erkämpft werden. Jacques Rancière spricht in diesem Sinne von den „Rechten derer, die nicht die Rechte haben, die sie haben und die die Rechte haben, die sie nicht haben". 38

Rancière befasst sich theoretisch näher mit den Prozessen, in welchen die Teilnahme von Personen an Politik als solcher in Frage steht und erstritten wird. Er stellt begrifflich Polizei und Politik gegenüber, wobei sein Begriff der Politik gerade diejenigen Situationen bezeichnet, , wenn die natürliche Ordnung der Herrschaft unterbrochen ist “ ${ }^{39}$ So ist in seinem Verständnis die politische Tätigkeit diejenige,

„die einen Körper von dem Ort entfernt, der ihm zugeordnet war oder die Bestimmung eines Ortes ändert; sie lässt sehen, was keinen Ort hatte, gesehen zu werden, lässt eine Rede hören, die nur als Lärm gehört wurde“. ${ }^{40}$

Diese Befassung mit den Momenten, in denen die Teilnahme selbst an Politik - im üblichen Verständnis - verhandelt wird, ist für das „Recht, Rechte zu haben“ bedeutsam. Hier richtet sich der Blick auf die äußerste Begründung des Rechts, als Rechtsträger:in anerkannt zu werden. Um Rechte politisch zu erkämpfen, um mit einem Menschenrechtsvokabular Gehör zu finden, ist ebenfalls ein Maß an Sichtbarkeit und Anerkennung als politische Person notwendig. Die Bedingungen, unter denen Menschen Rechte erstreiten, sind insofern selbst wiederum zu einem gewissen Grad rechtlich bedingt. Nicht nur sind die explizit vorgesehenen Möglichkeiten politischer Aktivität beispielsweise durch Versammlungs- und Vereinigungsfreiheit gerahmt. Sondern

\footnotetext{
${ }^{38}$ Rancière, Who is the Subject of the Rights of Man?, South Atlantic Quarterly 103 (2004), 297 (302) - eigene Übersetzung des Zitats aus dem Englischen. In der biblischen Geschichte der Töchter des Zelophehads findet sich ein ähnliches Motiv, wenn die bis dahin nicht erbberechtigten Töchter ihr Erbrecht einfordern und zugesprochen bekommen, Num. 27, 2-7.

${ }^{39}$ Rancière, Das Unvernehmen. Politik und Philosophie, 2002, 24.

${ }^{40}$ Ibid., 41.
} 
das Recht beeinflusst Voraussetzungen politischen Streits auch durch Regeln der Bewegungsfreiheit, des Zugangs zu Kommunikation oder sonstiger öffentlicher Sichtbarkeit.

Das „Recht, Rechte zu haben“ befindet sich insofern in einem ständigen Wechselspiel von Recht und Politik, wie sie auch Étienne Balibar in seiner Auslegung von Arendt unterstreicht. ${ }^{41}$ Einerseits können rechtliche Dokumente allein das „Recht, Rechte zu haben“" nicht endgültig absichern, wie der vorangegangene Abschnitt diskutiert hat. Diese Rechtsgarantien statuieren einzelne Rechte, sie können aber nicht verhindern, dass an den Rändern ihrer Anwendbarkeit oder durch Missachtung von Garantien Rechte ausgehöhlt werden und Menschen in Situationen von Rechtlosigkeit geraten. Andererseits finden Politiken der Rechte nicht in Ablösung vom Recht statt: Im Erstreiten und Begründen von Rechten spiegeln sich Ideen über ein besseres Recht oder eine bessere Interpretation von Gesetzen. ${ }^{42}$

Zunächst betont diese Linie der Interpretation also, dass das „Recht, Rechte zu haben" nicht in einer vorpolitischen Existenz, sondern in den Prozessen der aktiven Begründung von Rechten zu suchen ist. Es steht in diesem Sinne weder innerhalb noch außerhalb der rechtlichen Ordnung. ${ }^{43}$

Das „Recht, Rechte zu haben“ geht nicht sonstigen Rechten voraus, sondern bildet ein Recht, welches inmitten sämtlicher Rechtsgarantien immer wieder von Menschen gegenseitig abgesichert, geprüft und erneuert werden muss. In seiner Beschreibung, wie politische Gleichheit erstritten wird, verwendet Jacques Rancière das Bild einer Bühne, auf welcher Personen, die zuvor nicht als politisch Gleiche anerkannt waren, ihre Stimme zu Gehör bringen und sich als Gleiche zeigen. ${ }^{44}$ Dabei wird deutlich, wie es sich um ein Erkämpfen der Anerkennung als Rechtsträger:in handelt, und wie zugleich die Begründung von Rechten nicht einseitig zu erreichen ist, sondern der Anerkennung durch andere bedarf.

In verwandtem Sinn weist Judith Butler darauf hin, dass das „Recht, Rechte zu haben" bei Arendts selbst die Form einer Deklaration habe. ${ }^{45}$ Dass Arendt nach ihrer Kritik der Menschenrechte wiederum ein Recht einführt, sei gewissermaßen ein Ausdruck performativer Politik. Ayten Gündoğdu teilt diese Lesart und verbindet sie mit Arendts Konzeption von Freiheit nicht als innere Haltung oder gegebene Bedingungen, sondern als politische Praxis. ${ }^{46}$ Arendt schreibt: „Gleichheit ist nicht gegeben, und als Gleiche nur sind wir das Produkt menschlichen Handelns. Gleiche werden wir als Glieder einer Gruppe, in der wir uns kraft unserer eigenen Entscheidung Rechte gegenseitig garantieren. ${ }^{\text {(47 }}$

${ }^{41}$ Balibar, (De)Constructing the Human as Human Institution: A Reflection of the Coherence of Hannah Arendt's Practical Philosophy, Social Research 74/3 (2007), 731; ders., Citizenship, 2012, 65. Zum gleichrangigen Verhältnis von Recht und Politik in Arendts politischem Denken s. auch Volk, Arendtian Constitutionalism. Law, Politics and the Order of Freedom, 2015, 210.

${ }^{42}$ Vgl. auch Cover, Nomos and Narrative, Harvard Law Review 97/1 (1983), 47.

${ }^{43}$ Barbour, Between Politics and Law: Hannah Arendt and the Subject of Rights, in: Goldoni/ McCorkindale (Hrsg.), Hannah Arendt and the Law, 2013, 315.

${ }^{44}$ Rancière, Das Unvernehmen. Politik und Philosophie, 2002, 58.

${ }^{45}$ Butler/Chakravorty Spivak, Who Sings the Nation-state? Language, Politics, Belonging, 2007, 44.

${ }^{46}$ Gündoğdu, Rightlessness in an Age of Rights. Hannah Arendt and the Contemporary Struggles of Migrants, 2015, 171.

${ }^{47}$ Arendt, Elemente und Ursprünge totaler Herrschaft, 17. Aufl. 2014, 622. 


\section{Das „Rechte, Rechte zu haben““ in Konstellationen räumlicher Distanz}

Dass der Ausdruck des „Rechts, Rechte zu haben“ heute weiter als erkenntnisreich empfunden wird, liegt nach verschiedenen Interpretationen also daran, dass er den Scheinwerfer auf die Bedingungen richtet, in denen universelle Rechte begründet und garantiert werden. Das „Recht, Rechte zu haben“ ist insofern immer in der Schwebe. Der Ausdruck schärft die Aufmerksamkeit dafür, dass effektive Rechtsgarantien auf einem Minimum der politischen Anerkennung beruhen. Während rechtliche Garantien, insbesondere in Menschenrechtsabkommen, zwar das Projekt universeller Rechte vorantreiben, können sie nicht aus sich heraus verhindern, dass an Rändern der Anwendbarkeit oder in Lücken praktischer Wirksamkeit dennoch Rechtlosigkeit entsteht. Daneben trägt zur Absicherung des „Rechts, Rechte zu haben“ bei, dass Menschen Rechte erstreiten können - dies unterstreicht also die Bedeutung von politischer Sichtbarkeit und Räumen der politischen Verhandlung von Rechten.

Rancières Denken über politische Kämpfe wurde hinsichtlich der Situation von Migrierenden und Schutzsuchenden vielfach aufgegriffen. So fragt Samantha Velluti im Anschluss an Rancière, inwiefern der EU Kontext die rechtlichen und politischen Verfahren bereitstellt, in denen Asylsuchende ihre Gleichheit erstreiten können. ${ }^{48}$ Generell gibt es aus den vergangenen Jahren sehr einsichtsreiche Forschung zu Migration und politischen Kämpfen um Rechte. ${ }^{49}$

In der Frage, wie gerade für Flüchtlinge das „Recht, Rechte zu haben“ gesichert werden kann, ist ein wichtiger und wenig beleuchteter Aspekt jedoch die Rolle physischer Distanz. Viele der Situationen, in denen Menschen am Leben bedroht und dabei staatlichem Handeln ausgeliefert sind, finden gerade an territorialen Grenzen und hinsichtlich durch staatliches Handeln aufrecht erhaltener Distanz statt. Man denke an die oben genannten Beispiele der europäischen Grenzen: Menschen, die auf dem Mittelmeer nicht gerettet werden; Menschen, die abgefangen und nach Libyen zurückgebracht werden; Menschen, deren Visa abgelehnt werden, die ihnen eine Flucht nach Europa ermöglichen würden. In diesen und vielen anderen Fällen, stehen nicht nur fundamentale Rechte in Frage, sondern auch die Bedingungen, unter denen diese Rechte diskutiert und allenfalls erstritten werden können. Dabei spielt der physische Abstand gerade auch für das Verhandeln und Erstreiten von Rechten eine überragende Rolle. Er beeinflusst die juridischen Möglichkeiten, Rechte zu erstreiten. Er beeinflusst aber auch, wie diese Konstellationen politisch zur Aufmerksamkeit und Sprache gebracht werden können. Zwischen den Schutz- bzw. Rechtssuchenden und den verantwortlichen Öffentlichkeiten, welche diese Rechte anerkennen könnten, befindet sich eine räumliche Distanz, welche auch Mechanismen der Politik erschwert.

Wie aber beeinflusst dies das oben identifizierte Wechselspiel von Recht und Politik bei der immer wieder neuen Absicherung des „Rechts, Rechte zu haben“? Rancières Beschreibung des Ringens um Anerkennung als politisch sprechendes Subjekt ist dabei

${ }^{48}$ Velluti, Who has the Right to have Rights? The Judgments of the CJEU and the ECtHR as Building Blocks for a European ,ius commune“ in Asylum Law, in: Morano-Foadi/Vickers (Hrsg.), Fundamental Rights in the EU. A Matter for Two Courts, 2015, 139 (140).

${ }^{49}$ Z. B. McNevin, Ambivalence and Citizenship: Theorising the Political Claims of Irregular Migrants, Millennium: Journal of International Studies 41/2 (2013), 182; Schwiertz, Migration und radikale Demokratie. Politische Selbstorganisierung von migrantischen Jugendlichen in Deutschland und den USA, 2019; Stierl, Migrant Resistance in Contemporary Europe, 2018. 
interessant. Sein Bild der Bühne verdeutlicht die Schwierigkeiten, welche Konstellationen physischer Distanz erzeugen. Eine Bühne ist ein Ort, auf den sich Aufmerksamkeit richtet; es rückt Handlungen in den Fokus und verleiht ihnen Bedeutung. Es ist insofern ein hilfreiches Bild für politisches Handeln, mit welchem Menschen um Anerkennung als politische Subjekte und um Anerkennung als politische Gleiche ringen. Die Bühne ist nicht lediglich ein Ort der Verstärkung, sondern im Moment des Auftretens und Gesehenwerdens wandelt sich eine Handlung oder ein Sprechakt als solcher. Herausragende Momente des Betretens einer Bühne lassen sich in verschiedenen emanzipatorischen Bewegungen ausmachen. In den politischen Kämpfen der Französischen Revolution gehört dazu sicherlich das Assemblée Nationale, in dem sich die bis dahin nicht als zur politischen Mitbestimmung Berechtigten versammelten. Im Kampf gegen rassistische Segregation wurden die Sitze im Bus gewissermaßen zu Rosa Parks Bühne, auf der eine sonst alltägliche Handlung zum mutigen und einflussreichen Kampf um Gleichheit wurde.

Insofern sind die politischen Kämpfe um Rechte in Zusammenhang mit Migration verschieden. Diejenigen, die über Regeln der Migrationskontrolle entscheiden und daher Adressatinnen des Einforderns von Rechten sind, befinden sich nicht in der Nähe der Fordernden. Die um Rechte Kämpfenden mögen eine Bühne betreten, aber die Zuschauer sind nicht anwesend. Die Absicherung des „Rechts, Rechte zu haben“ stellt sich im Kontext von Migration komplizierter dar. Das Wechselspiel von Recht und Politik, welches die Existenz von Rechten bedingt, baut zu einem gewissen Grad auf die Annahme von Kopräsenz. Diejenigen aber, die durch Recht ferngehalten werden, sind unbedingt in der Lage, dem durch politisches Handeln etwas entgegenzusetzen.

In diesem Sinne hat Martina Tazzioli Rancières Fokus auf das öffentliche Erscheinen und Sprechen als ein „Bürger-Modell von Politik“ kritisiert und darauf hingewiesen, dass dieses Modell eine bestimmte politische Räumlichkeit voraussetzt. ${ }^{50}$ In der Tat stützt sich die Beschreibung auf Annahmen der Kopräsenz von Personen. Ist das ein Problem der Theorie, oder eher ein Ausdruck der Voraussetzungen von Politik, welche eben auf Begegnung beruht, und so besonders auch auf Begegnung in einem geteilten physischen Raum? Rancière selbst wirft die Frage von räumlicher Distanz auf, wenn er gegen Ende seines Buches schreibt:

„Eine politische Gemeinschaft ist nicht die Aktualisierung des gemeinsamen Wesens oder des Wesens des Gemeinsamen. Sie ist die Vergemeinschaftung dessen, was nicht als gemeinsam gegeben ist: zwischen Sichtbarem und Unsichtbarem, Nahem und Fernem, Anwesendem und Abwesendem." ${ }^{\text {51 }}$

Die Folgerungen sind für das „Recht, Rechte zu haben“ in Konstellationen räumlicher Distanz vielschichtig: Zum einen ist Sichtbarkeit heute nicht mehr nur an Kopräsenz geknüpft. Kommunikationsmedien und -technologien spielen eine erhebliche

\footnotetext{
${ }^{50}$ Tazzioli, Border interruptions and spatial disobediences beyond the scene of the political, Darkmatter 12 (2015), http://www.darkmatter101.org/site/2015/10/05/border-interruptions-and-spatial-di sobediences-beyond-the-scene-of-the-political/

${ }^{51}$ Rancière, Das Unvernehmen. Politik und Philosophie, 2002, 147.
} 
Rolle in Prozessen, in denen Rechtlosigkeit angeklagt wird..$^{52}$ Dennoch bleibt die Situation insofern verschieden, als dass für politische Kämpfe und Protest die physische Anwesenheit eine Rolle spielt. Wie es Arendt formuliert, ist das „Recht, Rechte zu haben“ bedroht gerade insofern als „der Platz in Welt" bedroht ist. Es geht, in anderen Worten, gerade um die Tatsache, das menschliche Existenz körperliche Existenz ist. Das Fordern von Rechten ist auf die Bedingungen dieser körperlichen Existenz bezogen, und so kommt der körperlichen Präsenz bei diesem Fordern eine Rolle für die Möglichkeiten performativer Politik zu. ${ }^{53}$

Schließlich verweist das Problem des „Rechts, Rechte zu haben“ in Konstellationen räumlicher Distanz auch wiederum auf die Bedeutung juridischer Verfahren. Rechtliche Festschreibungen allein können, wie oben festgestellt, nicht das „Recht, Rechte zu haben" garantieren, da sie immer ein Außen behalten und nicht gegen das Herausfallen aus dem eigenen Anwendungsbereich schützen können. Zugleich eröffnen menschenrechtliche Verfahren auch Räume, in denen der Universalitätsanspruch von Rechten verhandelt wird. Über die Auslegung von konkreten Normen erhält so der Anspruch des „Rechts, Rechte zu haben“ ebenfalls eine Bühne. Gerichtsverfahren sind insofern Orte öffentlicher Auseinandersetzung und Sichtbarmachung: Oftmals wird erst durch die Verhandlung eines konkreten Falls die Situation von systematischen Rechtsverletzungen einer größeren Öffentlichkeit bekannt. Zusammen mit den sie begleitenden Einschätzungen, Eingaben und Stellungnahmen werden internationale Verfahren oft zu einer Gelegenheit der nicht nur rechtlichen, sondern auch politischen Debatte über Rechte. $^{54}$

\section{Kopräsenz und die Territorialität von Rechten}

Letztlich wirft die Debatte um das „Recht, Rechte zu haben“ also auch die Frage nach der Rolle von Kopräsenz für Politik und Recht auf. Dieses Ergebnis muss auch in Zusammenhang mit sonstigen Entwicklungen betrachtet werden: wie verändert sich in Zeiten der Globalisierung das Verhältnis von Recht, Politik und räumlicher Anwesenheit?

Am Ausgangspunkt steht ein spezifischer Entwurf politischer Ordnung, bei dem Recht und Politikterritorial gerahmt sind. Diese Territorialität lässt sich insofern als universalistisch deuten, als die Kopräsenz an einem Ort als Kriterium politischer Gleichheit als ein auf Inklusion ausgerichtetes Kriterium verstanden wird. Demokratie meint in diesem Sinne die Herrschaft aller Anwesenden - aber die Institutionalisierung demokratischer Entscheidungsverfahren erfordert eine Abgrenzung der als Anwesenden gezählten, welche dann entlang territorialer Grenzen verläuft. Diese Idee von Kopräsenz als Grundlage politischer Gleichheit war natürlich zu aller Zeit nur ein normativer Horizont, niemals die Wirklichkeit. Politik beruht auf den sich aus zwischenmenschlicher Interaktion ergebenden Organisationsfragen. Dass insofern Politik grundsätzlich

\footnotetext{
${ }^{52}$ Das ist ganz praktisch zu erkennen an der herausragenden Bedeutung von Aufnahmen, mit denen Flüchtende Misshandlungen dokumentieren und medial teilen, für eine Verwendung solcher Dokumentation s. z. B. Keady-Tabbal/Mann, Tents at Sea: How Greek Officials Use Rescue Equipment for Illegal Deportations, Just Security, 22. Mai 2020, https://www.justsecurity.org/70309/tents-at-seahow-greek-officials-use-rescue-equipment-for-illegal-deportations/

${ }^{53}$ Vgl. Butler, Notes Towards a Performative Theory of Assembly, 2015, 45, 55

${ }^{54}$ Dazu auch in Schmalz, Newsletter Menschenrechte 2019/5, 375.
} 
zwischen denen stattfindet und auch institutionalisiert wird, die sich räumlich begegnen, ist vernünftig.

Zugleich sind zwischenmenschliche Zusammenhänge heute wesentlich vielfältiger als nur die sich aus räumlichem Zusammentreffen ergebenden. Einflüsse bestehen über Territorien hinaus, und mit der zunehmenden Digitalisierung sind auch die Möglichkeiten der Begegnung und geteilte kommunikative Räume nicht mehr gänzlich oder auch nur vorrangig an physischen Raum gekoppelt. Globalisierung hat weder räumliche Zusammenhänge als solche unwichtiger gemacht, noch ist die Bedeutung gänzlich gleichgeblieben. Stattdessen haben komplexe Verschiebungen und Überlagerungen stattgefunden. All das spiegelt sich auch im Recht, welches vielfach funktional den Handlungen folgt, die es regelt: des Datenverkehrs, des Handels, der Investitionen, der Kommunikation. Was folgt daraus für das gegenseitige Verhältnis von Recht und Politik? Dass die Transnationalisierung Fragen für demokratische Legitimation aufwirft, ist vielfach diskutiert worden. Die vervielfältigten Zusammenhänge werfen letztlich auch die Frage nach dem Raum von Politik, und eben nach der Begründung von Rechten auf. Ist Politik primär eine Frage zwischen den Kopräsenten? Wie gestaltet sich politische Gleichheit jenseits von Kopräsenz?

Im Kontext von Migration zeigen sich ganz konkret die fortdauernden Herausforderungen, so bezeichnete universelle Rechte auch tatsächlich jedem Menschen zu garantieren. In Konversation mit dem Universalitätsanspruch von Rechten tritt dabei immer wieder die Suche nach konkreten Zusammenhängen, welche die staatliche Verantwortung begründen. Dieses Element des Konkreten kann durchaus jenseits von Kopräsenz bestehen, es bleibt aber wohl insofern Grundlage von Rechtsbegründung, als Rechte wirksam eben zwischen Menschen sind. Dass der Ausdruck des „Rechts, Rechte zu haben" heute in einem solchen Maße zum Kristallisationspunkt von Debatten geworden ist, ist dabei selbst Ausdruck einer universalistischen Blickrichtung. Es hat, um der Argumentationslinie von Butler und Gündoğdu zu folgen, selbst den Charakter einer Deklaration, dass die Suche nach dem „Recht, Rechte zu haben“ noch nicht für beendet erklärt werden kann.

Dana Schmalz,

MPI für Ausländisches Öffentliches Recht und Völkerrecht, E-Mail: schmalz@mpil.de 